\title{
Topologically protected braiding in a single wire using Floquet Majorana modes
}

\author{
Bela Bauer, ${ }^{1}$ T. Pereg-Barnea, ${ }^{2,3}$ Torsten Karzig, ${ }^{1}$ Maria-Theresa Rieder, ${ }^{3}$ Gil Refael,,${ }^{4,5}$ Erez Berg, ${ }^{3,6}$ and Yuval Oreg ${ }^{3}$ \\ ${ }^{1}$ Station Q, Microsoft Corporation, Santa Barbara, California 93106, USA \\ ${ }^{2}$ Department of Physics, McGill University, Montréal, Québec, Canada \\ ${ }^{3}$ Department of Condensed Matter Physics, Weizmann Institute of Science, Rehovot 76100, Israel \\ ${ }^{4}$ Walter Burke Institute for Theoretical Physics and Institute for Quantum Information and Matter, \\ California Institute of Technology, Pasadena, California 91125, USA \\ ${ }^{5}$ Department of Physics, California Institute of Technology, Pasadena, California 91125, USA \\ ${ }^{6}$ Department of Physics, James Franck Institute, University of Chicago, Chicago, Illinois 60637, USA
}

(Received 1 November 2018; revised manuscript received 4 April 2019; published 3 July 2019)

\begin{abstract}
Majorana zero modes are a promising platform for topologically protected quantum information processing. Their non-Abelian nature, which is key for performing quantum gates, is most prominently exhibited through braiding. While originally formulated for two-dimensional systems, it has been shown that braiding can also be realized using one-dimensional wires by forming an essentially two-dimensional network. Here, we show that in driven systems far from equilibrium, one can do away with the second spatial dimension altogether by instead using quasienergy as the second dimension. To realize this, we use a Floquet topological superconductor which can exhibit Majorana modes at two special eigenvalues of the evolution operator, 0 and $\pi$, and thus can realize four Majorana modes in a single, driven quantum wire. We describe and numerically evaluate a protocol that realizes a topologically protected exchange of two Majorana zero modes in a single wire by adiabatically modulating the Floquet drive and using the $\pi$ modes as auxiliary degrees of freedom.
\end{abstract}

\section{DOI: 10.1103/PhysRevB.100.041102}

Nonequilibrium systems have recently been shown to host a variety of novel phenomena with no equilibrium system equivalent. One of the early examples was discussed in Ref. [1], which demonstrated that a driven $p$-wave superconducting wire can possess not only the well-known Majorana zero modes (MZMs) at zero energy [2,3], but also so-called Majorana $\pi$ modes (MPMs) at frequency $\omega / 2$, with $\omega$ the frequency of the external drive. These are but an example of a broader class of anomalous Floquet topological phases [4,5], with no analog in static (time-independent) systems. Other examples include Floquet symmetry-protected topological phases [6-9], and the closely related time crystals [8,10-14], where periodically driven interacting and disordered systems show a response at a multiple of the drive period. In all these systems, discrete time-translation symmetry protects novel quantum states.

It is natural to ask whether the topological degrees of freedom that emerge in driven systems can be used to supplement equilibrium topological phases. Particularly interesting are Majorana zero modes [15-19]. It is well known that they exhibit non-Abelian statistics: When several MZMs are present, the many-body ground state becomes degenerate, and adiabatically exchanging two well-separated MZMs carries out a nontrivial unitary transformation within the ground-state manifold [20,21]. Such braiding operations form the basis of topological quantum computation [22,23]. Physically, MZMs are realized as zero-energy excitations in one- [2,24-27] and two- [28-31] dimensional topological superconductors. While these systems are of great interest for quantum computing, non-Abelian braiding itself remains a tantalizing fundamental effect, and demonstrating it would be a tremendous breakthrough.
In the following, we show that MPMs emerging in driven systems allow for remarkable new braiding protocols, going beyond what is possible in equilibrium systems. Strictly speaking, braiding is only possible beyond one spatial dimension: two quasiparticles cannot be exchanged on a single wire while being distant from each other. In this work, however, we show that in periodically driven systems, quasienergy provides an additional synthetic dimension that can be used in conjunction with real space. Roughly speaking, the two kinds of Majorana states in Floquet superconductors-MZMs and MPMs-live a parallel existence at two different frequencies. As pointed out first in Ref. [1], they are precisely decoupled from each other as long as the drive is invariant under time translation by one period. It follows that half-frequency pulses can be used to couple the MZMs and MPMs [32].

References [33,34] also propose using a combination of MZMs and MPMs as well as half-frequency pulses to simulate braiding operations. However, the scheme we perform here is a nonlocal braid rather than the local operation at one end of the system proposed in Refs. [33,34]. The nonlocality of our scheme leads to topological protection, which is the essence of topological quantum computation, as it allows storing and processing quantum information in a way that is protected against all local noise sources.

Floquet braiding. We begin with a one-dimensional topological superconductor, which under a period- $T$ drive may enter a Floquet topological superconducting phase $[1,35]$. As a function of material and drive parameters, each edge of the system may have no MZMs, one MZM, and/or another Majorana mode with energy at the Floquet zone boundary. We denote this quasienergy by $\pi / T$ and refer to the corresponding Majorana mode as a Majorana $\pi$ mode (MPM). A 
time-periodic system only allows quasienergies inside the Floquet zone, $-\pi / T \leqslant \epsilon<\pi / T$. Therefore, particle-hole symmetry requires that Majorana modes come in pairs at all energies except zero and $\pi / T$, which is where unpaired Majorana modes can be found. Moreover, as long as time periodicity is conserved, the MZMs and MPMs do not hybridize even if their wave functions overlap in space. This property allows us to move them past each other and enables the procedure, which does not require any fine-tuning of the Hamiltonian or its time dependence [36].

There are several experimental schemes for MZM exchange. The simplest one is to physically move the MZMs [37]. Alternatively, consider a system made up of four MZMs at fixed locations, but with tunable interactions between them [38-41]; in this case, at any time during the braid two of the four Majorana modes are strongly coupled, but the dominant coupling is changed in a particular order to effectively perform a braid operation. Similarly, a sequence of 2-MZM measurements can be used to implement measurement-only variants of braiding [42-44]. In either case, at least two quantum wires are required.

Our proposed braiding protocol is most closely akin to an approach with four MZMs, of which two are coupled at any time. Our four states, however, are a pair of MZMs and another pair of MPMs. To introduce interactions between MZMs and MPMs, we apply a time-dependent perturbation in restricted regions, thus locally breaking the time-translation symmetry that protects the MPMs. We numerically confirm below that such a perturbation acts only locally even though timetranslation symmetry is a global symmetry. We then combine this with moving the MZMs and MPMs to achieve braiding.

Two-part drive model. Let us consider the Kitaev Hamiltonian:

$$
\begin{aligned}
H\left(\mu_{i}, w_{i}, \Delta_{i}\right)= & \sum_{i}\left[-\mu_{i} c_{i}^{\dagger} c_{i}-\frac{w_{i}}{2}\left(c_{i}^{\dagger} c_{i+1}+\text { H.c. }\right)\right] \\
& +\sum_{i} \frac{\Delta_{i}}{2}\left(c_{i} c_{i+1}+\text { H.c. }\right)
\end{aligned}
$$

and construct the Floquet operator with period $T$ :

$$
\begin{gathered}
U_{F}=e^{-i H_{0} T / 2} e^{-i H_{1} T / 2}, \\
H_{0}=H\left(\mu_{i}=0, w_{i}=2 \pi \lambda_{0} / T, \Delta_{i}=-2 \pi \lambda_{0} / T\right), \\
H_{1}=H\left(\mu_{i}=2 \pi \lambda_{1} / T, w_{i}=0, \Delta_{i}=0\right),
\end{gathered}
$$

where $H_{0}$ is the Hamiltonian of a Kitaev chain at the "sweet spot" of the topological phase (see below) and $H_{1}$ is the Hamiltonian of a trivial phase with only chemical potential. For couplings $\lambda_{0}, \lambda_{1} \in[0,1]$ ( $\hbar=1$ throughout), this gives rise to the phase diagram [12] (Fig. 1) with the four phases characterized by the presence or absence of MZMs and MPMs. Each phase contains a point of vanishing correlation length, aka "sweet spots," where the MZM and/or MPM states are localized on a single site. The sweet spots are indicated by the gray crosses in Fig. 1. These points are discussed in Sec. A of the Supplemental Material [45]. A convenient choice of parameters is given by that of the right panel of Fig. 1, where the superscript refers to the phases as follows: 1, trivial phase;

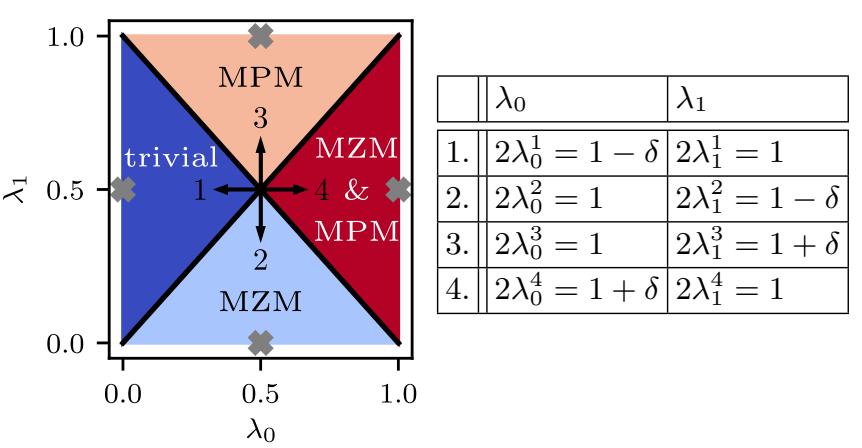

FIG. 1. Left: Phase diagram of the Floquet system in terms of the strength of the topological (trivial) Hamiltonian $H_{0}\left(H_{1}\right)$ in the two-part drive [see Eqs. (3) and (4)]. It is possible to realize four phases characterized by the presence of zero or $\pi$ modes: 1 , trivial; 2, MZMs; 3, MPMs; and 4, MZMs and MPMs. The gray crosses mark the sweet spots of the corresponding phases with vanishing correlation lengths. Right: Parametrization used in Eq. (2) to obtain the phase diagram. The parameter $\delta$ quantifies the distance to the critical point and the direction of increasing $\delta$ is indicated in the left panel.

2, MZM only; 3, MPM only; and 4, both MZMs and MPMs. The parameter $\delta$ quantifies the distance of all the phases to the critical point and is connected to the correlation length, with $\delta=0$ corresponding to the critical point and $\delta=1$ to the points with vanishing correlation length (the black arrows in the left panel of Fig. 1 indicate the direction of increasing $\delta$ ). Throughout, we consider $U_{F}$ to encode an elementary Floquet cycle with period $T$.

To implement the Floquet braiding protocol, consider an inhomogeneous system, where different regions are in different phases with the possibility to move phase boundaries. Let $\vec{p}$ be a vector whose elements $p_{i} \in\{1,2,3,4\}$ indicate that the parameters of the bond $i$ correspond to phase $p_{i}$. We can then generalize the Floquet drive of Eq. (2) to the inhomogeneous case:

$$
\begin{gathered}
U_{F}(\vec{p})=e^{-i H_{0}(\vec{p}) T / 2} e^{-i H_{1}(\vec{p}) T / 2}, \\
H_{0}(\vec{p})=H\left(\mu_{i}=0, w_{i}=2 \pi \lambda_{0}^{p_{i}} / T, \Delta_{i}=-2 \pi \lambda_{0}^{p_{i}} / T\right), \\
H_{1}(\vec{p})=H\left(\mu_{i}=2 \pi \lambda_{1}^{p_{i}} / T, w_{i}=0, \Delta_{i}=0\right) .
\end{gathered}
$$

In an inhomogeneous system, MZMs and MPMs also form at the interfaces between phases with different topological order. For example, half of the system could be in phase 2 (MZM), and the other half in phase 4 (MZM and MPM). In such a case, the MZMs will form at the end of the system, one MPM will form at one end of the system, and the other one in the middle of the system.

To move the spatial phase boundaries as a function of time, we interpolate between two different systems described by vectors $\vec{p}$ and $\vec{q}$ by continuously tuning a parameter $s \in$ $[0,1]$ and applying Floquet drives analogous to Eq. (5), but with $H_{0}=[1-f(s)] H_{0}(\vec{p})+f(s) H_{0}(\vec{q})$, and similarly for $H_{1}$. Here, $f(s)$ is a function with $f(0)=0$ and $f(1)=1$; in our simulations, we choose $f(s)=\sin (s \pi / 2)^{2}$. We evolve from $s=0$ to $s=1$ over $N_{s}$ time steps. For sufficiently large $N_{s}$, if the initial state of this operation is an eigenstate of 
$U_{F}(\vec{p})$, the final state will be an eigenstate of $U_{F}(\vec{q})$. This can be considered a version of adiabaticity for driven systems [46-48] and be understood by the formal relation between each $U_{F}$ to a Floquet Hamiltonian $H_{F}=i\left(\log U_{F}\right) / T$. The spectrum of $H_{F}$ corresponds to the quasienergy spectrum of the Floquet unitary. We can therefore relate the deformation from $U_{F}(\vec{p})$ to $U_{F}(\vec{q})$ to a deformation of the corresponding Floquet Hamiltonian from $H_{F}(\vec{p})$ to $H_{F}(\vec{q})$. The adiabatic condition can then be formulated with respect to the quasienergy spectrum of $H_{F}$. Dynamically changing the Floquet operator weakly breaks the time-translation symmetry that protects the MPMs similar to how energy conservation is broken in time-dependent equilibrium systems. To reduce the corresponding errors in the braiding protocol, we choose a smooth evolution that changes the Floquet operator in each cycle and strongly suppresses the $\pi / T$ components as $N_{s}$ becomes large except for the desired local perturbations discussed below.

As a final ingredient to our protocol, we need to be able to couple nearby MZMs and MPMs. To explicitly introduce such a coupling, we insert a perturbation operator $U_{\text {pert }}$ after two elementary Floquet cycles. The coupling can be understood by considering that eigenvectors corresponding to quasienergies 0 and $\pi / T$ in $U_{F}$ all correspond to quasienergy 0 in $U_{F}^{2}$, and are therefore susceptible to perturbations. The effect of the perturbation is independent of the details as long as the perturbation is sufficiently generic; for our specific choice, see Sec. B of the Supplemental Material. The strength of the perturbation is quantified by a coupling strength $\lambda_{\text {pert }}$. Importantly, if $U_{\text {pert }}$ acts only in a specific region of the system, it will only couple a pair of nearby MZMs and MPMs in that region while leaving the ones far away unperturbed. We numerically probe this fact, which amounts to locally breaking time-translation symmetry, in the Supplemental Material.

Braiding protocol. We now turn to the full braid protocol. We start and end in a configuration where the entire system is in the regular, undriven, Kitaev phase, exhibiting MZMs at the system's edge. This allows state preparation in an undriven system. We then turn on the Floquet drive to perform a braid operation by following the steps in Fig. 2. Since all the Floquet-drive phases (2) are gapped around the respective 0 or $\pi$ modes, and the protocol never drives extended regions of the system through the phase transition at once, the Floquet quasienergy spectrum at each step of the evolution remains gapped. Therefore adiabaticity is maintained even in the thermodynamic limit by choosing $N_{s}$ which interpolates the move of the phase boundary by one site sufficiently large.

Throughout the evolution, the system contains at least a pair of MZMs, and, at intermediate stages, an additional pair of MPMs. In the case where both MZMs and MPMs and hence a total of four modes are present, we need to fix which pair encodes the quantum information. To achieve this, we apply a local time-translation-symmetry-breaking perturbation in a region in the middle of the system. Therefore, when both an MZM and an MPM are in the middle, they are split to finite energy and only two low-energy modes remain, which thus carry the encoded quantum state. When three modes, e.g., two MPMs and an MZM, are in the perturbed regime, one mode (which is a linear combination of the three modes) remains unperturbed while two are split away to finite energy. This
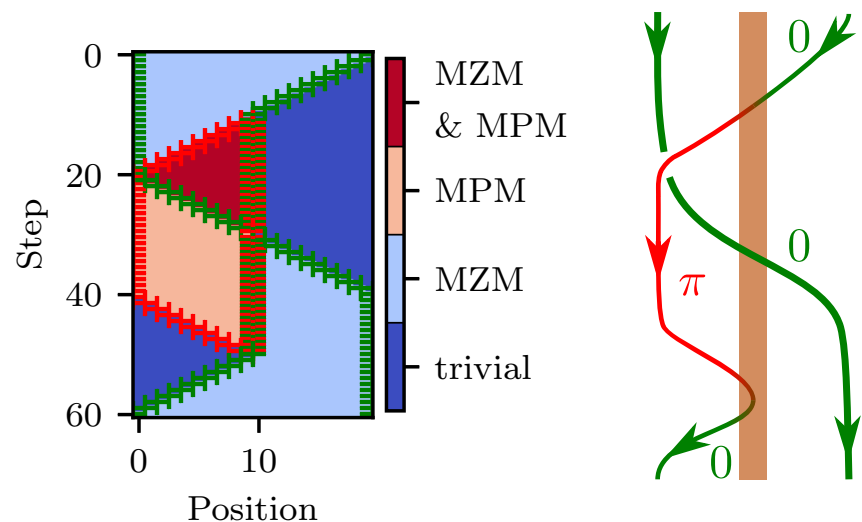

FIG. 2. Left: Full braid protocol for a system of $L=20$ sites. The colors correspond to different phases; green (red) crosses indicate the locations of MZMs (MPMs). Right: Schematic representation of the braiding process of two MZMs. In the center region it is possible to convert between MZMs (denoted by 0 ) and MPMs (denoted by $\pi$ ). After the right MZM has been converted into an MPM it can be safely moved past the left MZM in the region where time-translational symmetry is preserved.

enables us to effectively convert a MZM to a MPM mode and vice versa as indicated in Fig. 2.

Numerical results. A numerical implementation of the dynamical braiding is summarized in Fig. 3. Since the Hamiltonian is quadratic, the evolution of operators of the form $\vec{v} \cdot \vec{\gamma}$, where $\vec{\gamma}$ is a vector of Majorana operators such that $2 c_{i}=\gamma_{2 i-1}+i \gamma_{2 i}$, can be represented by an orthogonal matrix $\bar{U}$. Over the entire process, $\vec{v} \cdot \vec{\gamma}$ evolves into $(\bar{U} \vec{v}) \cdot \vec{\gamma}$ (see Sec. C of the Supplemental Material for details).

To define the relevant error measures, let $\gamma_{1,2}=\vec{v}_{1,2} \cdot \vec{\gamma}$ be initial (and final) MZMs. Then, we compute the $2 \times 2$

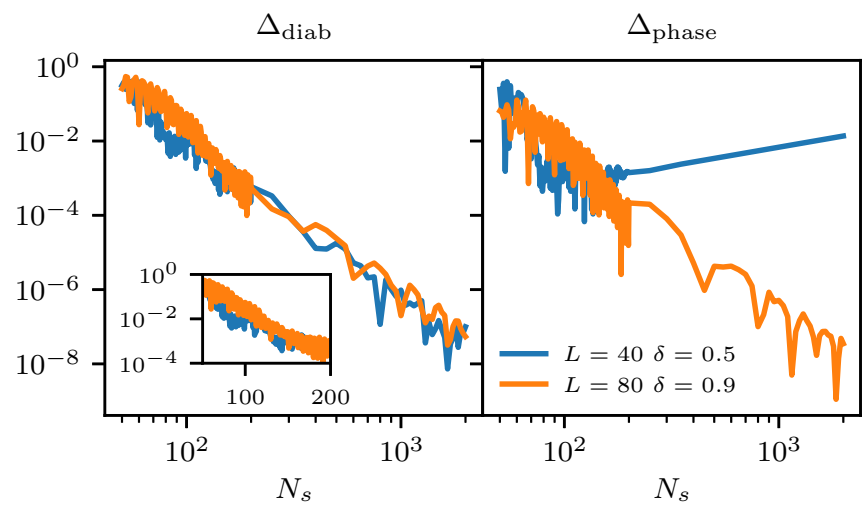

FIG. 3. Errors in the braid protocol, measured by the deviation from unitarity of the evolution in the low-energy subspace (left panel) and deviation in the applied phase (right panel; see main text for definitions of $\Delta_{\text {diab }}$ and $\Delta_{\text {phase }}$ ) as a function of the number of interpolation steps between stages of the protocol. In the limit of $N_{s} \rightarrow \infty$, adiabaticity is recovered. The errors generally vanish with a lower law; however, for fast protocols $\left(N_{s}<200\right)$ an exponential transient behavior is observed. In the phase error, the dependence on $N_{s}$ is nonmonotonic: for sufficiently slow protocols, the evolution becomes adiabatic with respect to the residual finite-size splitting of Majorana modes. Parameters used are $\lambda_{\text {pert }}=0.2$. 
matrix $\left(U_{r}\right)_{\alpha, \beta}=\vec{v}_{\alpha}^{T} \bar{U} \vec{v}_{\beta}(\alpha, \beta=1,2)$, which encapsulates how the entire time evolution acts on the low-energy Majorana subspace. In the ideal limit, $U_{r}=i \sigma^{y}$, where $\sigma^{y}$ denotes the usual Pauli matrix. We quantify deviations from this using two measures: $\Delta_{\text {diab }}=\left|U_{r}^{\dagger} U_{r}-1\right|$ captures deviations from unitarity, in particular diabatic corrections that excite fermions from the low-energy subspace to the excited states. Secondly, we compute the two eigenvalues of $U_{r}$ as $\left(r_{1} e^{i \phi_{1}}, r_{2} e^{i \phi_{2}}\right)$. In the ideal case, we expect $r_{1}=r_{2}=1$ and $\phi_{1}=-\pi / 2, \phi_{2}=$ $\pi / 2$. We define deviations from this as $\Delta_{\text {phase }}=\left|\phi_{1}+\pi / 2\right|+$ $\left|\phi_{2}-\pi / 2\right|$, where we sort eigenvalues such that $\phi_{1} \geqslant \phi_{2}$. Both measures are chosen to be independent of the basis choice for the Majorana subpsace since it is not unique in the case when they are exactly degenerate.

Figure 3 shows that increasing $N_{s}$ to perform a slower protocol improves the errors. At short times, the accuracy improves exponentially, while at long times a power-law behavior is observed, consistent with the nonanalytic time dependence of the driving Hamiltonian. Interestingly, the two error measures can exhibit qualitatively different behavior, as shown in the long-time behavior for $L=40, \delta=0.5$ : while the diabatic corrections continue to decrease, the error in the applied phase reaches a minimum value beyond which it increases again. This occurs because very slow protocols resolve the splitting of the low-energy manifold. For larger system sizes, such as $L=80$ and $\delta=0.9$, this crossover would occur at much slower protocol times $\left(\right.$ larger $N_{s}$ ). In most relevant parameter regimes, the error is dominated by diabatic corrections and not finite-size corrections, i.e., the error is independent of system size for all but the smallest systems. Details of the dependence of $\Delta_{\text {diab }}$ on other parameters such as $\delta$ and $\lambda_{\text {pert }}$ can be found in Sec. D of the Supplemental Material.

Topological protection and outlook. To conclude, we discuss in what sense braiding as described here is topologically protected. Just as many other new phenomena in periodically driven systems, MPMs are protected by time-translation symmetry. Therefore, braiding of MPMs is topologically protected only if no processes that break the periodicity of the drive are present. A subtle issue is that the braid process itself breaks time-translation symmetry and thus gives rise to dynamical corrections, but as we have shown above these can be systematically suppressed by adiabatically changing the drive parameters. Similar diabatic errors may also occur in the braiding of MZMs if operations are performed away from the adiabatic limit [49-60].
Importantly, unlike other symmetries that can give rise to multiple MZMs in a single wire, our Floquet approach does not require careful tuning of the instantaneous Hamiltonian. Thus it is much more experimentally accessible. We provide a perspective toward such realizations in systems based on superconducting quantum dot chains [61-63] in the Supplemental Material, where in particular we discuss a model that is able to implement the same behavior but requires timedependent control of only a single parameter (see Sec. E of the Supplemental Material). Perhaps the simplest realization, however, would be using a quantum wire proximity coupled to two superconductors, one grounded, and the other at a finite voltage. The ac Josephson effect gives rise to the time dependence leading to MPMs [64].

An important caveat is that we relied on the absence of heating. While this assumption is appropriate for the noninteracting limit, it is well known that driven interacting systems generically heat to infinite temperature [65-67]. However, there are known mechanisms such as many-body localization [67-70] as well as the prethermalization [71-78] which can be used to avoid heating and stabilize the results discussed here. While the details of this interacting scenario are an interesting open question left to future work, we note that a quantum dot chain would be particularly promising to reach the prethermal regime. As outlined in the Supplemental Material, control over two parameters (e.g., hopping and chemical potential) of the quantum dot chain is sufficient to reach the sweet spot regime of all four different phases (see Sec. F of the Supplemental Material). Close to the sweet spots, the bands of the continuum states become flat and are thus expected to show prethermalization.

Acknowledgments. This work was supported by NSERC DG (T.P.-B.), the BSF and ISF grants and by the European Research Council under the European Union's Seventh Framework Programme (FP7/2007-2013)/ERC Grant Agreement MUNATOP No. 340210, and under the European Union's Horizon 2020 Research and Innovation Programme (Grant Agreement LEGOTOP No. 788715) (Y.O.). Y.O. and E.B. acknowledge support from CRC 183 of the Deutsche Forschungsgemeinschaft. G.R. is grateful for support from the Institute of Quantum Information and Matter, an NSF frontier center with support from the Gordon and Betty Moore Foundation, as well as the Packard Foundation. We are also grateful for the hospitality of the Aspen Center for Physics, which is supported by National Science Foundation Grant No. PHY-1607761, and where part of the work was done.
[1] L. Jiang, T. Kitagawa, J. Alicea, A. R. Akhmerov, D. Pekker, G. Refael, J. I. Cirac, E. Demler, M. D. Lukin, and P. Zoller, Phys. Rev. Lett. 106, 220402 (2011).

[2] A. Kitaev, Phys. Usp. 44, 131 (2001).

[3] O. Motrunich, K. Damle, and D. A. Huse, Phys. Rev. B 63, 224204 (2001).

[4] T. Kitagawa, E. Berg, M. Rudner, and E. Demler, Phys. Rev. B 82, 235114 (2010).

[5] M. S. Rudner, N. H. Lindner, E. Berg, and M. Levin, Phys. Rev. X 3, 031005 (2013).

[6] C. W. von Keyserlingk and S. L. Sondhi, Phys. Rev. B 93, 245145 (2016).
[7] D. V. Else and C. Nayak, Phys. Rev. B 93, 201103(R) (2016).

[8] A. C. Potter, T. Morimoto, and A. Vishwanath, Phys. Rev. X 6, 041001 (2016).

[9] R. Roy and F. Harper, Phys. Rev. B 94, 125105 (2016).

[10] F. Wilczek, Phys. Rev. Lett. 109, 160401 (2012).

[11] A. Shapere and F. Wilczek, Phys. Rev. Lett. 109, 160402 (2012).

[12] V. Khemani, A. Lazarides, R. Moessner, and S. L. Sondhi, Phys. Rev. Lett. 116, 250401 (2016).

[13] D. V. Else, B. Bauer, and C. Nayak, Phys. Rev. Lett. 117, 090402 (2016). 
[14] C. W. von Keyserlingk, V. Khemani, and S. L. Sondhi, Phys. Rev. B 94, 085112 (2016).

[15] J. Alicea, Rep. Prog. Phys. 75, 076501 (2012).

[16] C. Beenakker, Annu. Rev. Condens. Matter Phys. 4, 113 (2013).

[17] S. D. Sarma, M. Freedman, and C. Nayak, npj Quantum Inf. 1, 15001 (2015).

[18] R. M. Lutchyn, E. P. A. M. Bakkers, L. P. Kouwenhoven, P. Krogstrup, C. M. Marcus, and Y. Oreg, Nat. Rev. Mater. 3, 52 (2018).

[19] R. Aguado, Riv. Nuovo Cimento 40, 523 (2017).

[20] D. A. Ivanov, Phys. Rev. Lett. 86, 268 (2001).

[21] A. Stern, F. von Oppen, and E. Mariani, Phys. Rev. B 70, 205338 (2004).

[22] A. Y. Kitaev, Ann. Phys. (NY) 303, 2 (2003).

[23] C. Nayak, S. H. Simon, A. Stern, M. Freedman, and S. Das Sarma, Rev. Mod. Phys. 80, 1083 (2008).

[24] R. M. Lutchyn, J. D. Sau, and S. Das Sarma, Phys. Rev. Lett. 105, 077001 (2010).

[25] Y. Oreg, G. Refael, and F. von Oppen, Phys. Rev. Lett. 105, 177002 (2010).

[26] V. Mourik, K. Zuo, S. M. Frolov, S. R. Plissard, E. P. A. M. Bakkers, and L. P. Kouwenhoven, Science 336, 1003 (2012).

[27] S. Nadj-Perge, I. K. Drozdov, J. Li, H. Chen, S. Jeon, J. Seo, A. H. MacDonald, B. A. Bernevig, and A. Yazdani, Science 346, 602 (2014).

[28] M. Sato and S. Fujimoto, Phys. Rev. B 79, 094504 (2009).

[29] P. A. Lee, arXiv:0907.2681.

[30] L. Fu and C. L. Kane, Phys. Rev. Lett. 100, 096407 (2008).

[31] J. D. Sau, R. M. Lutchyn, S. Tewari, and S. Das Sarma, Phys. Rev. Lett. 104, 040502 (2010).

[32] This was previously discussed in the dual language of destroying the emerging $\mathbb{Z}_{2}$ symmetry in an Ising time crystal in, e.g., Ref. [79].

[33] R. W. Bomantara and J. Gong, Phys. Rev. Lett. 120, 230405 (2018).

[34] R. W. Bomantara and J. Gong, Phys. Rev. B 98, 165421 (2018).

[35] D. E. Liu, A. Levchenko, and H. U. Baranger, Phys. Rev. Lett. 111, 047002 (2013).

[36] For a protocol that relies on fine-tuning of the Hamiltonian, see Ref. [80].

[37] J. Alicea, Y. Oreg, G. Refael, F. von Oppen, and M. P. A. Fisher, Nat. Phys. 7, 412 (2011).

[38] B. van Heck, A. R. Akhmerov, F. Hassler, M. Burrello, and C. W. J. Beenakker, New J. Phys. 14, 035019 (2012).

[39] T. Hyart, B. van Heck, I. C. Fulga, M. Burrello, A. R. Akhmerov, and C. W. J. Beenakker, Phys. Rev. B 88, 035121 (2013).

[40] D. Aasen, M. Hell, R. V. Mishmash, A. Higginbotham, J. Danon, M. Leijnse, T. S. Jespersen, J. A. Folk, C. M. Marcus, K. Flensberg, and J. Alicea, Phys. Rev. X 6, 031016 (2016).

[41] T. Karzig, Y. Oreg, G. Refael, and M. H. Freedman, Phys. Rev. X 6, 031019 (2016).

[42] P. Bonderson, M. Freedman, and C. Nayak, Phys. Rev. Lett. 101, 010501 (2008).

[43] S. Plugge, A. Rasmussen, R. Egger, and K. Flensberg, New J. Phys. 19, 012001 (2017).

[44] T. Karzig, C. Knapp, R. M. Lutchyn, P. Bonderson, M. B. Hastings, C. Nayak, J. Alicea, K. Flensberg, S. Plugge, Y. Oreg, C. M. Marcus, and M. H. Freedman, Phys. Rev. B 95, 235305 (2017).
[45] See Supplemental Material at http://link.aps.org/supplemental/ 10.1103/PhysRevB.100.041102 for an overview, as well as Refs. [60,81-83].

[46] H. Breuer and M. Holthaus, Phys. Lett. A 140, 507 (1989).

[47] T. Kitagawa, T. Oka, A. Brataas, L. Fu, and E. Demler, Phys. Rev. B 84, 235108 (2011).

[48] P. Weinberg, M. Bukov, L. D’Alessio, A. Polkovnikov, S. Vajna, and M. Kolodrubetz, Phys. Rep. 688, 1 (2017).

[49] T. Karzig, G. Refael, and F. von Oppen, Phys. Rev. X 3, 041017 (2013).

[50] M. S. Scheurer and A. Shnirman, Phys. Rev. B 88, 064515 (2013).

[51] C. S. Amorim, K. Ebihara, A. Yamakage, Y. Tanaka, and M. Sato, Phys. Rev. B 91, 174305 (2015).

[52] T. Karzig, A. Rahmani, F. von Oppen, and G. Refael, Phys. Rev. B 91, 201404(R) (2015).

[53] T. Karzig, F. Pientka, G. Refael, and F. von Oppen, Phys. Rev. B 91, 201102(R) (2015).

[54] F. L. Pedrocchi and D. P. DiVincenzo, Phys. Rev. Lett. 115, 120402 (2015).

[55] F. L. Pedrocchi, N. E. Bonesteel, and D. P. DiVincenzo, Phys. Rev. B 92, 115441 (2015).

[56] C. Knapp, M. Zaletel, D. E. Liu, M. Cheng, P. Bonderson, and C. Nayak, Phys. Rev. X 6, 041003 (2016).

[57] M. Hell, J. Danon, K. Flensberg, and M. Leijnse, Phys. Rev. B 94, 035424 (2016).

[58] M. Sekania, S. Plugge, M. Greiter, R. Thomale, and P. Schmitteckert, Phys. Rev. B 96, 094307 (2017).

[59] A. Rahmani, B. Seradjeh, and M. Franz, Phys. Rev. B 96, 075158 (2017).

[60] B. Bauer, T. Karzig, R. V. Mishmash, A. E. Antipov, and J. Alicea, SciPost Phys. 5, 004 (2018).

[61] J. D. Sau and S. D. Sarma, Nat. Commun. 3, 964 (2012).

[62] I. C. Fulga, A. Haim, A. R. Akhmerov, and Y. Oreg, New J. Phys. 15, 045020 (2013).

[63] Z. Su, A. B. Tacla, M. Hocevar, D. Car, S. R. Plissard, E. P. A. M. Bakkers, A. J. Daley, D. Pekker, and S. M. Frolov, Nat. Commun. 8, 585 (2017).

[64] Y. Peng and G. Refael, Phys. Rev. B 98, 220509 (2018).

[65] L. D'Alessio and M. Rigol, Phys. Rev. X 4, 041048 (2014).

[66] A. Lazarides, A. Das, and R. Moessner, Phys. Rev. E 90, 012110 (2014).

[67] P. Ponte, A. Chandran, Z. Papić, and D. A. Abanin, Ann. Phys. (NY) 353, 196 (2015).

[68] D. A. Abanin, W. D. Roeck, and F. Huveneers, Ann. Phys. (NY) 372, 1 (2016).

[69] P. Ponte, Z. Papić, F. Huveneers, and D. A. Abanin, Phys. Rev. Lett. 114, 140401 (2015).

[70] A. Lazarides, A. Das, and R. Moessner, Phys. Rev. Lett. 115, 030402 (2015).

[71] D. A. Abanin, W. De Roeck, and F. Huveneers, Phys. Rev. Lett. 115, 256803 (2015).

[72] D. A. Abanin, W. De Roeck, W. W. Ho, and F. Huveneers, Phys. Rev. B 95, 014112 (2017).

[73] D. Abanin, W. De Roeck, F. Huveneers, and W. W. Ho, Commun. Math. Phys. 354, 809 (2017).

[74] T. Kuwahara, T. Mori, and K. Saito, Ann. Phys. (NY) 367, 96 (2016).

[75] T. Mori, T. Kuwahara, and K. Saito, Phys. Rev. Lett. 116, 120401 (2016). 
[76] M. Bukov, S. Gopalakrishnan, M. Knap, and E. Demler, Phys. Rev. Lett. 115, 205301 (2015).

[77] E. Canovi, M. Kollar, and M. Eckstein, Phys. Rev. E 93, 012130 (2016).

[78] M. Bukov, M. Heyl, D. A. Huse, and A. Polkovnikov, Phys. Rev. B 93, 155132 (2016).

[79] D. V. Else, B. Bauer, and C. Nayak, Phys. Rev. X 7, 011026 (2017).
[80] C.-K. Chiu, M. M. Vazifeh, and M. Franz, Europhys. Lett. 110, 10001 (2015).

[81] M. Wimmer, ACM Trans. Mathe. Software (TOMS) 38, 30 (2012).

[82] S. Bravyi and D. Gosset, Commun. Math. Phys. 356, 451 (2017).

[83] A. Auerbach, Interacting Electrons and Quantum Magnetism (Springer Science \& Business Media, 1994). 